\title{
Untargeted analysis of plasma samples from pre-eclamptic women reveals polar and apolar changes in the metabolome
}

\author{
Katrin N. Sander ${ }^{1,3} \cdot$ Dong-Hyun Kim ${ }^{3} \cdot$ Catharine A. Ortori $^{3} \cdot$ Averil Y. Warren $^{1} \cdot$ Uchenna C. Anyanwagu $^{1}$. \\ Daniel P. Hay ${ }^{1} \cdot$ Fiona Broughton Pipkin ${ }^{2} \cdot$ Raheela N. Khan ${ }^{1}$ (D) David A. Barrett ${ }^{3}$
}

Received: 20 May 2019 / Accepted: 27 September 2019 / Published online: 27 November 2019

(c) The Author(s) 2019

\begin{abstract}
Introduction Pre-eclampsia is a hypertensive gestational disorder that affects approximately 5\% of all pregnancies.

Objectives As the pathophysiological processes of pre-eclampsia are still uncertain, the present case-control study explored underlying metabolic processes characterising this disease.

Methods Maternal peripheral plasma samples were collected from pre-eclamptic $(n=32)$ and healthy pregnant women $(\mathrm{n}=35)$ in the third trimester. After extraction, high-resolution mass spectrometry-based untargeted metabolomics was used to profile polar and apolar metabolites and the resulting data were analysed via uni- and multivariate statistical approaches. Results The study demonstrated that the metabolome undergoes substantial changes in pre-eclamptic women. Amongst the most discriminative metabolites were hydroxyhexacosanoic acid, diacylglycerols, glycerophosphoinositols, nicotinamide adenine dinucleotide metabolites, bile acids and products of amino acid metabolism.

Conclusions The putatively identified compounds provide sources for novel hypotheses to help understanding of the underlying biochemical pathology of pre-eclampsia.
\end{abstract}

Keywords Pregnancy, human $\cdot$ Pre-eclampsia $\cdot$ Metabolomics $\cdot$ Metabolic profiling

\begin{tabular}{ll}
\multicolumn{2}{l}{ Abbreviations } \\
CV-ANOVA & ANOVA of the cross-validated residuals \\
FDR & False discovery rate \\
LC-MS & Liquid chromatography-mass spectrometry \\
MVA & Multivariate analysis \\
OPLS-DA & $\begin{array}{l}\text { Orthogonal projections to latent structures } \\
\text { discriminant analysis }\end{array}$ \\
PCA & Principle component analysis \\
PET & Pre-eclampsia (pre-eclamptic toxaemia)
\end{tabular}

Electronic supplementary material The online version of this article (https://doi.org/10.1007/s11306-019-1600-8) contains supplementary material, which is available to authorized users.

Raheela N. Khan

raheela.khan@nottingham.ac.uk

1 Division of Medical Science and Graduate Entry Medicine, School of Medicine, University of Nottingham, Royal Derby Hospital, Uttoxeter Road, Derby DE22 3DT, UK

2 Division of Child Health, Obstetrics \& Gynaecology, School of Medicine, University of Nottingham, City Hospital, Nottingham NG5 1PB, UK

3 Centre for Analytical Bioscience, School of Pharmacy, University of Nottingham, Nottingham NG7 2RD, UK
QC Quality control

ROC Receiver operator characteristic

RSD Relative standard deviation

UVA Univariate analysis

VIP Variable influence on projection

\section{Introduction}

Pre-eclampsia is a syndrome that complicates between 3 and $5 \%$ of pregnancies (Lisonkova and Joseph 2013). It is defined by de novo hypertension with proteinuria and directly implicated in $14 \%$ of global maternal deaths, preterm deliveries and infants of low-birthweight (Steegers et al. 2010; WHO 2015). The impact of this disease is far-reaching with a significantly increased risk of diabetes and cardiovascular disease in later life (Murphy and Smith 2016).

The pathophysiology of pre-eclampsia has been the subject of substantial research efforts in past decades (Huppertz 2008). There is a general consensus that the maternal preeclamptic syndrome is caused by maternal systemic endothelial dysfunction triggered by pregnancy (Redman 1991). In the first stage, inadequate villous trophoblast differentiation 
is seen as the origin of pre-eclampsia. The second (symptomatic) stage proposes a state in which the mother, due to genetic or environmental factors or increased placental mass, is not able to cope with the placental release of apoptotic microvesicles (Huppertz 2008).

Insufficient insight into the pathophysiology of preeclampsia is a major reason for the lack of early diagnostic markers, hampering the reliable diagnosis or evaluation of disease progression essential for the management of this potentially life-threatening gestational complication. Therefore, increasing efforts aim to understand perturbed metabolic pathways, both as potential sources of early biomarkers and to uncover metabolic processes involved in the pathophysiology of pre-eclampsia. In this context, the discovery of early biomarkers is an important aim for diagnostic purposes. However, an examination of the metabolome during the clinical phase of pre-eclampsia is also crucial to understand ongoing changes in metabolic processes during the symptomatic phase of the disease. Most of the previously suggested biomarkers for pre-eclampsia are proteins, presumed to be derived from the placenta or damaged vascular endothelium (Wu et al. 2015). Evaluation of their sensitivity and specificity was inconclusive with more studies needed to confirm their utility in the clinic.

A number of authors have previously searched for biomarkers in maternal blood in pre-eclampsia using metabolomics. These studies assessed maternal plasma (BahadoSingh et al. 2012; De Oliveira et al. 2012; Kenny et al. 2005, 2008; Kenny et al. 2010; Turner et al. 2008) or serum (Bahado-Singh et al. 2013; Kuc et al. 2014; Odibo et al. 2011) collected before (first or second trimester) (BahadoSingh et al. 2012, 2013; Kenny et al. 2010; Kuc et al. 2014; Odibo et al. 2011) and/or after (De Oliveira et al. 2012; Kenny et al. 2005, 2008; Turner et al. 2008) disease onset. Half of the studies distinguished between early and late onset pre-eclampsia (Bahado-Singh et al. 2012, 2013; De Oliveira et al. 2012; Kuc et al. 2014), the other half used sample sets without considering the gestation at the onset of the disease (Kenny et al. 2005, 2008, 2010; Odibo et al. 2011; Turner et al. 2008). Analyses were performed with sample numbers ranging from 8 to 100 in the pre-eclampsia group (BahadoSingh et al. 2012, 2013; De Oliveira et al. 2012; Kenny et al. 2005, 2008, 2010; Kuc et al. 2014; Odibo et al. 2011; Turner et al. 2008). A range of metabolites including polar and apolar compounds have been proposed as biomarkers. The most comprehensive study in this context utilised a validated untargeted analysis using LC-MS with approximately 100 patients in each subject group (Kenny et al. 2010): It was suggested that a combination of 14 metabolites could predict pre-eclampsia before the onset of the disease.

However, a recent systematic review and meta-analysis concluded that "although there are multiple potential biomarkers for PE their efficacy has been inconsistent", (Wu et al. 2015). This confusion is likely to be caused by the different experimental (chemical and instrumental) approaches and clinical designs chosen and highlights the need for additional studies in this field. Confirmation of a specific metabolic pathway would set the basis for future studies focussing on specific substance groups.

Although early markers are important, metabolic changes are most likely to be maximal after onset of the disease. As a prelude to the application of metabolomics in identifying metabolites associated with the pre-eclamptic syndrome, the present study was designed to test plasma from women already diagnosed with pre-eclampsia to provide proof of concept that the metabolome of pre-eclamptic women is distinct from that of women with a normal uncomplicated pregnancy. For this, a comprehensive analytical approach was chosen to avoid focussing on a specific group of metabolites.

\section{Materials and methods}

\subsection{Chemicals}

Acetonitrile, chloroform and ammonium acetate were purchased from Fisher Scientific (Loughborough, UK). Ammonium carbonate, 2-propanol and methanol were purchased from Fluka (Sigma-Aldrich, Hannover, Germany). Deionized water (18.2 M $\Omega$ ) was prepared using an ELGA USFMaxima water purification system (Marlow, UK).

\subsection{Plasma collection and preparation}

Maternal peripheral blood samples were taken at Royal Derby Hospital (UK) from healthy $(\mathrm{n}=35)$ or pre-eclamptic $(n=32)$ pregnant women after obtaining fully informed written consent. Ethics approval for the sample collection and utilisation was granted by Derbyshire Research Ethics Committee (REC Reference No. 09/H0401/90). Recruited subjects in the pre-eclampsia group had (1) normal booking blood pressures (at 12-20 weeks of pregnancy), (2) subsequently developed blood pressures of $\geq 140$ systolic or $\geq 90$ diastolic on two occasions (minimum $24 \mathrm{~h}$ apart) and (3) had at least $1+$ proteinuria $(\geq 300 \mathrm{mg} / \mathrm{L}$ ) using dipstick analysis (Meyer et al. 1994). These criteria correspond to current guidelines for diagnosis of pre-eclampsia (Magee et al. 2014). Subjects in the control group did not have any documented hypertensive problems throughout their pregnancy. Pre-eclampsia and control samples were matched for age, BMI, systolic booking blood pressure, gestational age at sampling, parity and ethnicity. Patients were not matched for diastolic booking blood pressure, gestational age at delivery, birth weight/customized weight centile of the baby or multiple pregnancy. Patient demo-graphics are shown in 
Table 1. Early onset pre-eclampsia affected 10 of the 32 pre-eclampsia patients.

\subsection{Plasma preparation}

Blood samples were taken into BD Vacutainer EDTA blood collection tubes and centrifuged at $1000 \times g$ for $15 \mathrm{~min}$ at $4{ }^{\circ} \mathrm{C}$. The plasma was transferred to Eppendorf tubes in $0.5 \mathrm{~mL}$ aliquots, snap-frozen and stored at $-80{ }^{\circ} \mathrm{C}$. Only samples that were processed within $1 \mathrm{~h}$ were included in this study.

\subsection{Metabolite extraction}

Polar and apolar metabolites were extracted from plasma samples $(400 \mu \mathrm{L})$ with $500 \mu \mathrm{L}$ chloroform:methanol (1:2) followed by centrifugation at $13,000 \times g$ for $10 \mathrm{~min}$ at $4{ }^{\circ} \mathrm{C}$. $75 \mu \mathrm{L}$ of the lower chloroform (apolar) phase were added to prepared vials containing $1500 \mu \mathrm{L}$ isopropanol, giving the readily injectable apolar fraction. $600 \mu \mathrm{L}$ of the aqueous (polar) supernatant were transferred into Eppendorf tubes and mixed with $900 \mu \mathrm{L}$ acetonitrile to precipitate proteins. Samples were centrifuged at $13,000 \times g$ for $10 \mathrm{~min}$ at $4{ }^{\circ} \mathrm{C}$ and $1400 \mu \mathrm{L}$ were transferred into Eppendorf tubes.
Following solvent evaporation, the residue was resuspended in $100 \mu \mathrm{L}$ starting mobile phase $\left(\mathrm{H}_{2} \mathrm{O}\right.$ :acetonitrile 4:6) prior to injection. A pooled quality control (QC) sample was prepared for each fraction. This was achieved by pooling a $10 \mu \mathrm{L}$ aliquot of each extract of the respective fraction.

\subsection{LC-MS polar fraction}

The LC-MS method was based on a previously reported method (Kim et al. 2014). $10 \mu \mathrm{L}$ of the polar fraction of each sample were injected onto an Exactive high-resolution mass spectrometry (HRMS) system operating in positive and negative ion mode (Accela U-HPLC system coupled to a HESI-II Exactive Orbitrap mass spectrometer, Thermo Fisher, Hemel Hempstead, UK). Chromatographic separation was achieved using a Sequant ZIC-pHILIC $5 \mu \mathrm{m}$ PEEK $150 \times 4.6 \mathrm{~mm}$ column (Merck Millipore, Darmstadt, Germany) using a gradient over 15 min (mobile phase A: $0 \min 40 \%$; 9 min $95 \%$; $10 \min 40 \% ; 15 \min 40 \%$ at $300 \mu \mathrm{L} /$ min). Mobile phase A was $\mathrm{H}_{2} \mathrm{O}$ with $20 \mathrm{mM}\left(\mathrm{NH}_{4}\right)_{2} \mathrm{CO}_{3}$ and mobile phase $\mathrm{B}$ consisted of acetonitrile without additive. Following interface parameters were used: spray voltage $4500 \mathrm{~V}$ (positive mode)/3500 V (negative mode); capillary temperature $275^{\circ} \mathrm{C}$; heater temperature $150^{\circ} \mathrm{C}$; flow rates of
Table 1 Patient demographics for maternal plasma collected from control and pre-eclampsia cohort

\begin{tabular}{|c|c|c|c|c|}
\hline & & $\begin{array}{l}\text { Control } \\
(n=35)\end{array}$ & $\begin{array}{l}\text { Pre-eclampsia } \\
(\mathrm{n}=32)\end{array}$ & $\mathrm{p}$ value \\
\hline Age (yrs) & & $28.9(6.7)$ & $30.2(5.1)$ & $>0.05$ \\
\hline BMI & & $27.0(7.0)$ & $28.0(5.1)$ & $>0.05$ \\
\hline Booking blood pressure systolic ( $\mathrm{mmHg}$ ) & & $109.4(8.4)$ & $112.6(9.1)$ & $>0.05$ \\
\hline Booking blood pressure diastolic $(\mathrm{mmHg})$ & & $63.6(7.4)$ & $69.8(8.7)$ & 0.003 \\
\hline Gestational age at sampling (wks) & & $36.7(3.2)$ & $35.9(2.8)$ & $>0.05$ \\
\hline Gestational age at delivery (wks) & & $38.3(2.0)$ & $36.9(2.6)$ & 0.016 \\
\hline Birth weight baby (g) & & $3149.1(698.9)$ & $2543.6(830.7)$ & 0.002 \\
\hline Customised weight centile baby & & $48.2(30.0)$ & $27.9(31.0)$ & 0.008 \\
\hline \multirow[t]{2}{*}{ Multiple pregnancy } & Singleton & $35(100 \%)$ & $28(87.5 \%)$ & 0.031 \\
\hline & Twins & 0 & $4(12.5 \%)$ & \\
\hline \multirow[t]{5}{*}{ Parity } & 0 & $14(40.0 \%)$ & $22(68.8 \%)$ & $>0.05$ \\
\hline & 1 & $13(37.1 \%)$ & $5(15.6 \%)$ & \\
\hline & 2 & $4(11.4 \%)$ & $4(12.5 \%)$ & \\
\hline & 3 & $3(8.6 \%)$ & $1(3.1 \%)$ & \\
\hline & 4 & $1(2.9 \%)$ & $0(0.0 \%)$ & \\
\hline \multirow[t]{4}{*}{ Ethnicity } & Africa & $0(0.0 \%)$ & $1(3.1 \%)$ & $>0.05$ \\
\hline & India/Pakistan & $4(11.4 \%)$ & $4(12.5 \%)$ & \\
\hline & Middle East & $0(0.0 \%)$ & $1(3.1 \%)$ & \\
\hline & $\begin{array}{l}\text { Southern/ } \\
\text { Northern } \\
\text { Europe }\end{array}$ & $31(88.6 \%)$ & $26(81.3 \%)$ & \\
\hline
\end{tabular}

Table shows mean (standard deviation) or numbers (\%) of $\mathrm{n}$ recruited patients. yrs years, wks weeks. Customised weight centiles were calculated using Weight Centile Calculator from GROW software version 6.7.7, 2016 (Gardosi et al. 1992, 1995). Significance testing was performed using student t-test (continuous variables) or Chi square test (categorical variables) 
sheath, auxiliary and sweep gas were 40, 5 and 1 (arbitrary units); capillary, tube and skimmer voltages were $-28.6 \mathrm{~V}$, $-67.5 \mathrm{~V}$ and $-16.6 \mathrm{~V}$. Data acquisition was performed in full scan mode with a range from $m / z, 70$ to 1400 .

\subsection{LC-MS apolar fraction}

The LC-MS/MS method was based on a previously reported method (Ravipati et al. 2015). $10 \mu \mathrm{L}$ of the apolar fraction of each sample were injected onto an Exactive HRMS system operating in positive and negative ion mode. Chromatographic separation was achieved using a Poroshell SBC18 $50 \times 2.1 \mathrm{~mm} 2.7 \mu \mathrm{m}$ column (Agilent, Santa Clara, US) using a gradient over $12 \mathrm{~min}$ (mobile phase B: $0 \mathrm{~min}$ $32 \%$; 1 min 60\%; 5 min 75\%; 6 min 100\%; 10 min 100\%; $11 \min 32 \% ; 12 \min 32 \%$ at $450 \mu \mathrm{L} / \mathrm{min})$. Mobile phase A consisted of $40 \%$ acetonitrile and $60 \% \mathrm{H}_{2} \mathrm{O}$ with $13 \mathrm{mM}$ $\mathrm{NH}_{4}$ Ac. Mobile phase B consisted of $10 \%$ acetonitrile, $10 \%$ $\mathrm{H}_{2} \mathrm{O}$ and $80 \%$ isopropanol with $13 \mathrm{mM} \mathrm{NH}_{4} \mathrm{Ac}$. Following interface parameters were used in both negative and positive mode: spray voltage $4000 \mathrm{~V}$; capillary temperature $250{ }^{\circ} \mathrm{C}$; heater temperature $300{ }^{\circ} \mathrm{C}$; flow rates of sheath, auxiliary and sweep gas were 30,15 and 5 (arbitrary units); capillary, tube and skimmer voltages were $-26.0 \mathrm{~V},-137.0 \mathrm{~V}$ and $-26.6 \mathrm{~V}$. Data acquisition was performed in full scan mode with a range from $\mathrm{m} / \mathrm{z}, 100$ to 1900 .

\subsection{LC-MS analysis}

Samples for polar and apolar fraction were analysed in separate runs according to a run design as suggested previously with QC and samples analysed after every 11 samples (Zelena et al. 2009). Samples were randomized before extraction and before injection. Pooled QC samples were used to assess the analytical performance.

\subsection{LC-MS data analysis}

Generated raw data was acquired using Xcalibur software (Thermo Scientific, Hemel Hempstead, UK). The data was pre-processed using Progenesis QI software (Nonlinear Dynamics, Newcastle upon Tyne, UK). Peak picking was performed with auto threshold and an automatically chosen QC injection served as reference for chromatographic alignment and normalisation. Peak intensities were normalised to all compounds. For this, abundance ratios between each sample and the reference run are calculated for all compounds. Log10 ratio distributions of each sample are then centred onto the $\log 10$ ratio distribution of the reference run by applying an additive or subtractive shift in the log space (Waters Corporation 2015). For further advanced multivariate analysis (MVA), the four datasets were merged to one large dataset before importing to SIMCA (Version
13.0, Umetrics AB, Umea, Sweden). The data was scaled in the default setting unit variance (UV) and log transformation was applied. PCA and OPLS-DA models of the data were created. Cross validation was performed by creating an OPLS-DA model with a portion of the observations to avoid potential bias. The remaining observations were subsequently fitted into this model. In the resulting model, variables with a VIP value $>1$ were identified as potential biomarkers.

Univariate analysis (UVA) was performed using Progenesis QI. Criteria for potential biomarkers were a QC $\mathrm{CV} \leq 30 \%$, a fold intergroup change of $\geq 1.5$ and significantly changed metabolite levels after $t$ test and correction for multiple testing using FDR (false discovery rate). Regression analysis was performed to evaluate the effects of two unmatched patient parameters (diastolic booking blood pressure and singleton/multiple pregnancy) as potential confounders. Other unmatched patient parameters (gestational age at delivery and birth weight/customized weight centile of the baby) were not tested as these parameters have a causal relationship with pre-eclampsia and with that do not meet the criteria for a confounding factor.

Biomarkers that were filtered by both UVA and MVA were putatively identified by searching for their $\mathrm{m} / \mathrm{z}$ values in the Human Metabolome Database (www.hmdb. ca), METLIN Metabolomics Database (www.metlin.scrip ps.edu) and Lipid Maps (www.lipidmaps.org). Putative identifications were based on isotope similarity $>75 \%$, mass error $<1.5 \mathrm{ppm}$ and expected or demonstrated presence in the human organism. Metabolite identification confidence was classified using identification classes (Sumner et al. 2007). Additionally, the quantitative identification score was determined as proposed previously (Sumner et al. 2014).

\section{Results}

\subsection{Metabolomic analysis of plasma from control and pre-eclamptic subjects}

Plasma samples from pregnant women with $(\mathrm{n}=32)$ and without $(n=35)$ pre-eclampsia were analysed in this study to search for metabolites indicating altered biological pathways involved in the pathophysiology of pre-eclampsia.

LC-MS metabolomics quality control (QC) samples met the required acceptability threshold for both the polar and apolar metabolomics analysis methods used in the study (Chan et al. 2011; Dunn et al. 2011). Assessment of the peak areas of key ions of QC samples confirmed analytical stability in terms of peak area $(<30 \%$ relative standard deviation (RSD)) and retention time ( $<2 \% \mathrm{RSD})$.

Initial processing of the metabolomics data from the study samples revealed 4379 peaks of polar compounds 
and 3153 peaks of apolar compounds detected by LC-MS. MVA and UVA of these data sets was used to define a set of metabolites which showed significant differences between the control and pre-eclampsia study groups.

MVA showed no visible separation of control and preeclampsia subjects in a principal component analysis (PCA) (data not shown). However, the orthogonal projections to latent structures discriminant analysis (OPLS-DA) model in Fig. 1a shows a complete separation of control subjects and pre-eclampsia subjects. The R2X(cum)-value of 0.286 and Q2(cum)-value of 0.514 are typical for clinical samples. Furthermore, the CV-ANOVA $p$ value is highly significant $\left(p=6 \times 10^{-7}\right)$, indicating a good model. Validation of the dataset was performed by randomly choosing $30 \%$ of the initial observations and creating a model with the remaining observations. The new model based on these training and prediction data sets is shown in Fig. 1b. Class affiliation of previously removed observations was then predicted. $85 \%$ of the observations were predicted correctly with a sensitivity of $82 \%$ and specificity of $89 \%$. This demonstrated good quality of the OPLS-DA training model, as a prediction of those observations that were left out would not be correct in an over-fitted model. Figure 1c shows a receiver operator characteristic (ROC) curve to evaluate the predictability of the OPLS-DA model shown in Fig. 1b (Eng 2007). The area under the curve was 0.922 . (a)

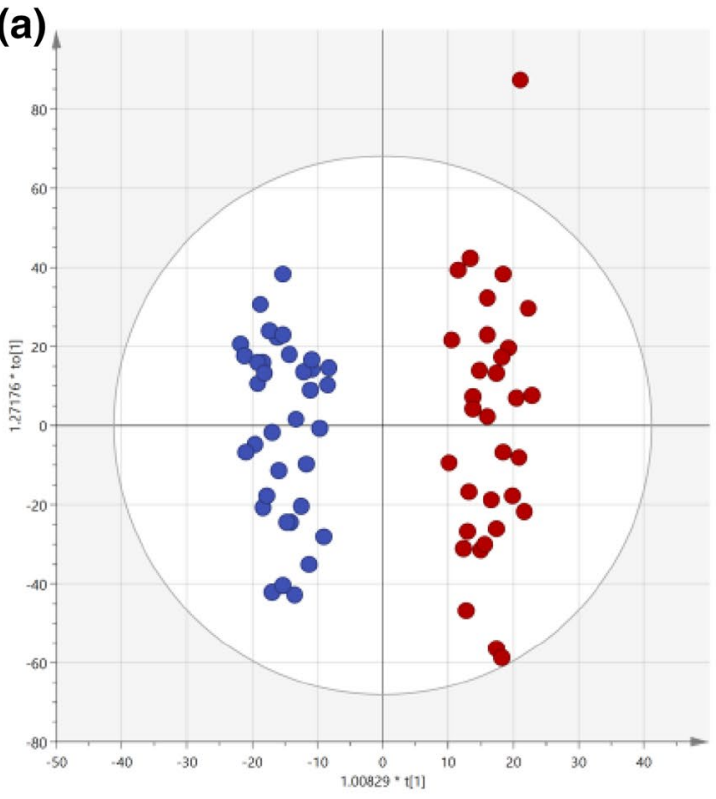

(b)

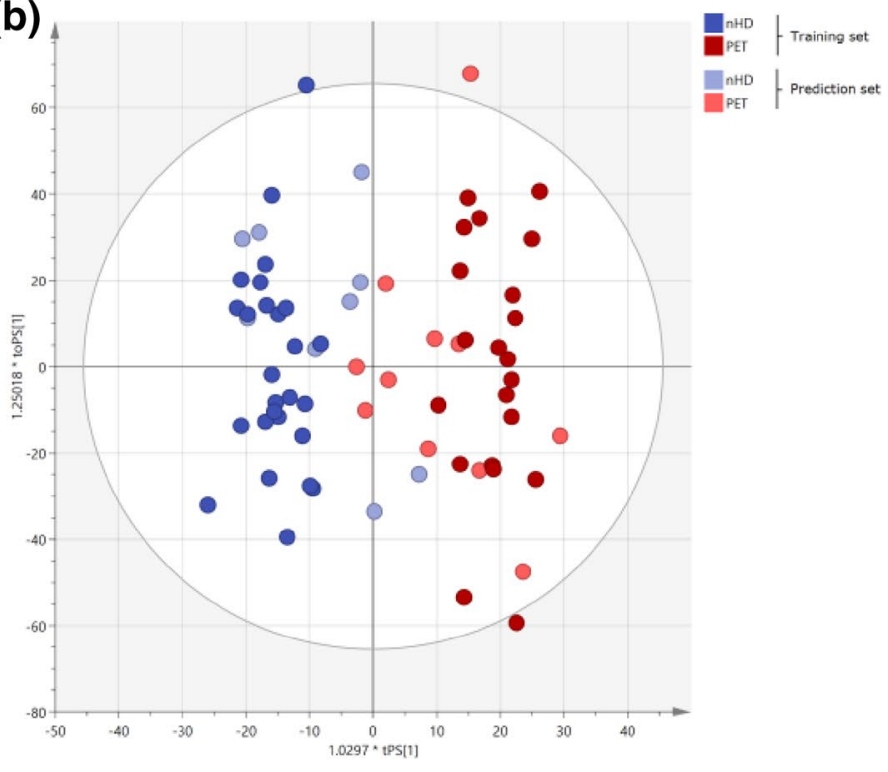

(c) ROC curve

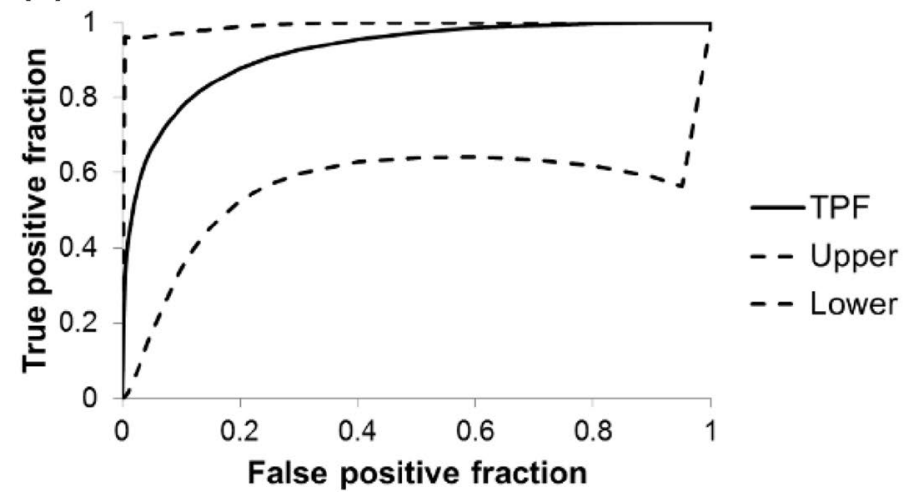

Fig. 1 Multivariate analysis based on all detected ions: a OPLSDA score plot of control (no hypertensive disease, $n H D, n=35$ ) and pre-eclampsia (PET, $\mathrm{n}=32$ ) samples for all variables. b OPLS-DA score plot of training and prediction set for control (no hypertensive disease, nHD) and pre-eclampsia (PET) samples for all variables. c
Receiver operator characteristic (ROC) curves for all variables. The figure shows the true positive fraction (TPF) with upper and lower $95 \%$ confidence intervals. The AUC is 0.922 with a standard error of 0.06 


\subsection{Putative plasma biomarkers of pre-eclampsia}

To identify biomarkers for pre-eclampsia, detected compounds were further filtered. Univariate analysis (UVA) yielded 37 metabolites after significance testing using $\mathrm{t}$-test and correction for multiple testing. Based on a variable influence on projection (VIP) value $>1$ in the cross validated OPLS-DA model shown in Fig. 1b, 2527 ions were filtered using multivariate analysis (MVA). All 37 compounds filtered by UVA were also filtered by MVA and are therefore promising candidates for biomarkers (see full list in the Supplemental data Table 1).

Two of the unmatched patient parameters were classified as potential confounders: (1) diastolic booking blood pressure and (2) singleton/multiple pregnancy. Regression analysis showed that none of these parameters were statistically significant (independent) confounders (data

(a)
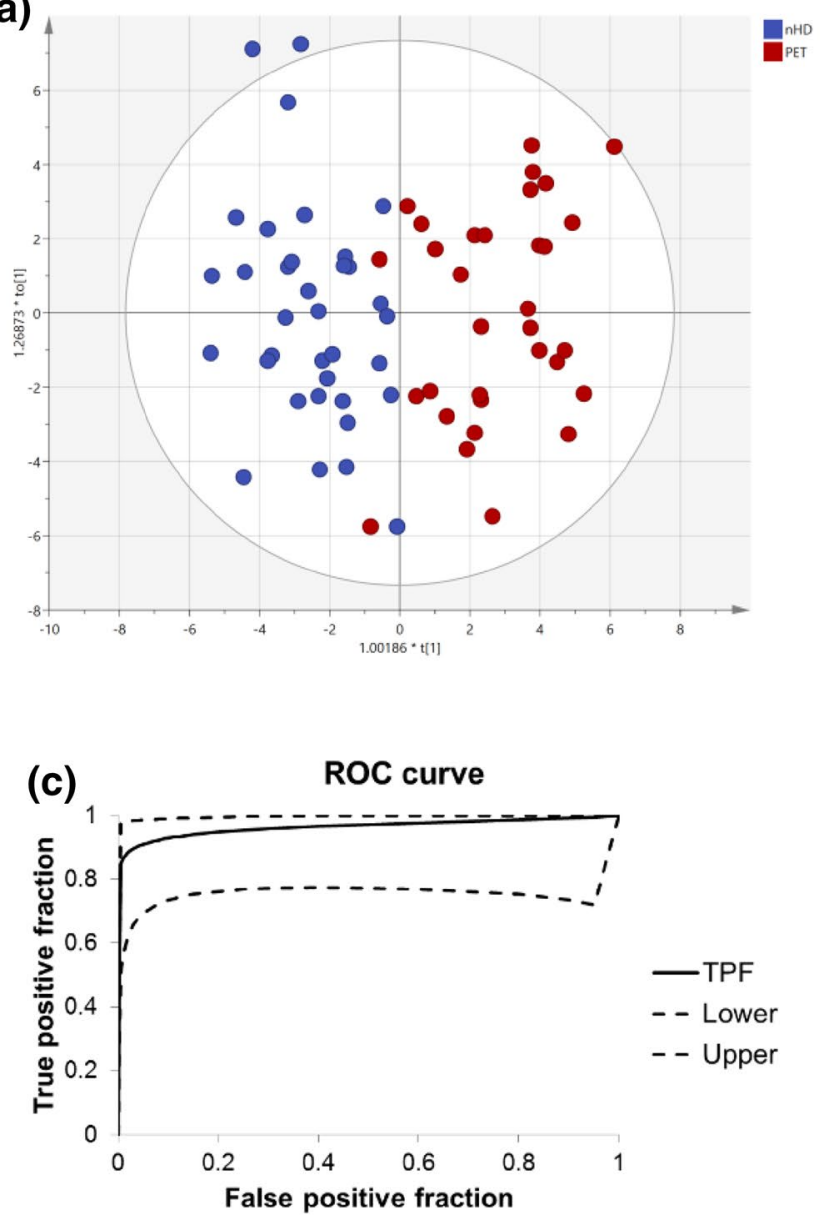

Fig. 2 Multivariate analysis based on the 35 most predictive ions: a OPLS-DA score plot of control (no hypertensive disease, nHD, $\mathrm{n}=35$ ) and pre-eclampsia (PET, $\mathrm{n}=32$ ) samples for the 35 most predictive ions. b OPLS-DA score plots of training and prediction set for control (no hypertensive disease, nHD) and pre-eclampsia (PET) not shown). Therefore, the data was not adjusted for these parameters.

Multivariate analysis was repeated based on the putative biomarker metabolites. Two compounds were removed from this list and all further analyses, as these were putatively identified as labetalol and terbutaline-medications commonly given to pre-eclamptic women. The PCA based on the 35 most predictive ions showed a visible separation of control and pre-eclampsia subjects (data not shown). Similarly, the OPLS-DA model in Fig. 2a shows a complete separation of control subjects and pre-eclampsia subjects. The R2X(cum)-value of 0.629, Q2(cum)-value of 0.648 and a highly significant CV-ANOVA p value $\left(\mathrm{p}=5.5 \times 10^{-12}\right)$ indicate an excellent model. A validation of the dataset was performed by randomly choosing $50 \%$ of the initial observations and creating a model with the remaining observations. The new model based on these training and prediction data

(b)
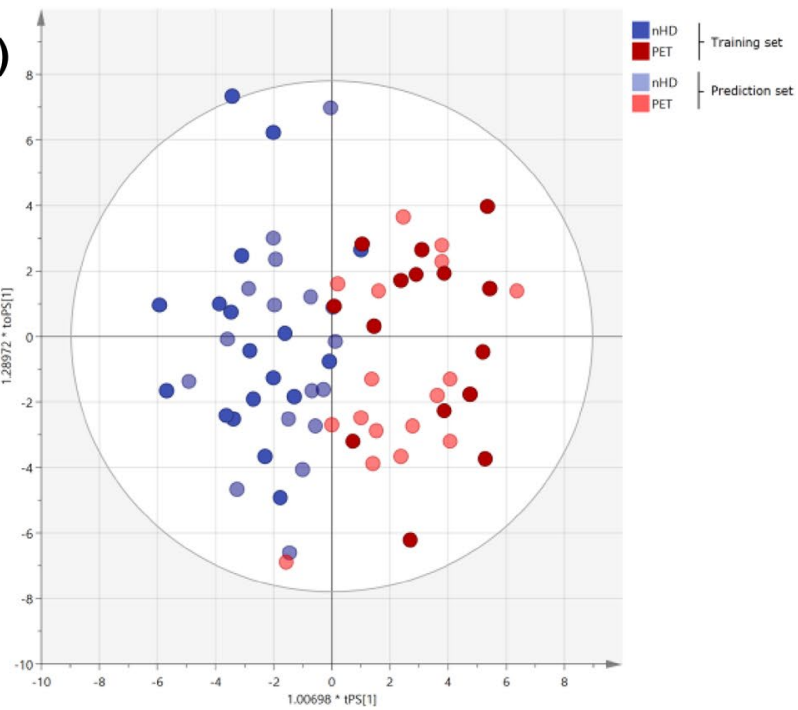

samples for the 35 most predictive ions. c Receiver operator characteristic (ROC) curves for the 35 most predictive ions. The figure shows the true positive fraction (TPF) with upper and lower $95 \%$ confidence intervals. The AUC is 0.964 with a standard error of 0.04 
sets is shown in Fig. 2b. The class affiliation of previously removed observations was then predicted. $91 \%$ of the observations were predicted correctly with a sensitivity of $82 \%$ and specificity of $100 \%$, demonstrating excellent quality of the OPLS-DA training model. Figure $2 \mathrm{c}$ shows a ROC curve to evaluate the predictability of the OPLS-DA model shown in Fig. 2b (Eng 2007). The area under the curve was 0.964 .

Using metabolomics databases, 9 of the 35 compounds could be putatively identified by compound class or unique assignment (see Supplemental data Tables 2 and 3): A taurodeoxycholic acid isomer, methionine sulfoxide, 3-hydroxyanthranilic acid, an N1-methyl-pyridone-carboxamide isomer and urocanic acid in the polar fraction and a hydroxyhexacosanoic acid isomer, two different types of diacylglycerols and a glycerophosphoinositol in the apolar fraction. Scatter plots of control vs PET normalised intensities for these compounds are shown in Fig. 3. Identification classes (Sumner et al. 2007) are shown in Supplemental data Tables 2 and 3 . The quantitative identification score was 1.0 in all cases (Sumner et al. 2014).

\section{Discussion}

The present study examined the metabolome of maternal plasma samples to identify altered metabolite pathways that may provide new insights into the underlying biology of pre-eclampsia. This is the first report of a separate analysis of polar and apolar metabolites in plasma samples for a metabolomics approach in this disorder. This comprehensive analytical approach leads to an improved resolution of the metabolome into its distinct components resulting in maximum coverage of the metabolome in the biological sample.

This study included patients with mixed risk factors for pre-eclampsia. The sample set for pre-eclampsia included subjects of mixed parity, women with early and late onset pre-eclampsia as well as single and multiple pregnancies. Parity and multiple pregnancy are important risk factors for pre-eclampsia. Furthermore early and late onset pre-eclampsia are increasingly seen as two different disease entities (Raymond and Peterson 2011). The biomarkers identified in this pilot work are therefore mainly informative for ongoing processes during the clinical appearance of the pre-eclamptic syndrome, which is a common feature of pre-eclampsia independent of its origin.

A total of 35 metabolites were found to significantly contribute to metabolic differences in plasma from pre-eclamptic and healthy pregnant women. Not all of these could be assigned chemical identities, but the use of database matching allowed putative identification of several metabolites.

The two N-methylpyridone carboxamide isomers are NAD metabolites and have previously been shown to accumulate in patients with renal failure (Rutkowski et al. 2003).
Levels were increased in plasma from pre-eclamptic women. They are of interest given that pre-eclampsia is characterised by proteinuria, an important diagnostic marker for underlying renal dysfunction. Metabolic markers may be superior to proteinuria as a diagnostic tool, as the reliability of the latter is under scrutiny (Magee et al. 2014).

A further set of metabolites showing increased levels in our pre-eclampsia study was assigned to three closely related bile acids: tauro-deoxycholic acid, taurourso-deoxycholic acid and taurocheno-deoxycholic acid. Taurine, an element of the three structural isomers, has previously been found as a biomarker in other studies analysing maternal blood in pre-eclampsia (Kenny et al. 2010; Kuc et al. 2014).

A previously proposed marker for oxidative stress, methionine sulfoxide, was increased in pre-eclampsia (Mashima et al. 2003; Raff et al. 2008). This is in line with the increased placental oxidative stress as a known feature in pre-eclampsia. Interestingly, levels of the antioxidant 3-hydroxyanthranilic acid were also raised in pre-eclamptic subjects. It is an intermediate of tryptophan catabolism via the kynurenine pathway, which is an important pathway for the synthesis of NAD. The kynurenine pathway including 3-hydroxyanthranilic acid as a therapeutic target in preeclampsia was previously hypothesised but further evidence is required (Worton et al. 2019). An earlier study looking at kynurenine pathway metabolites identified kynurenic acid as a plasma marker for pre-eclampsia, however, in contrast to our study no significant changes were seen for 3-hydroxyanthranilic acid (Nilsen et al. 2012).

Levels of the histidine metabolite urocanic acid were found to be elevated in plasma from pre-eclamptic subjects. Urocanic acid is mainly known for its role as photoprotectant in the skin, however, it was also identified in amniotic fluid from normal pregnancies (Orczyk-Pawilowicz et al. 2016).

Concentrations of several lipids were found to change significantly when comparing the control and pre-eclampsia group. The concentration of the oxylipin, hydroxyhexacosanoic acid, was found to be decreased in plasma from preeclamptic women. Its dehydroxylated form, hexacosanoic acid, was earlier listed as biomarker in pre-eclampsia (Kenny et al. 2010). The complex changes in the oxylipin metabolism are likely to cause substantial physiological effects considering the potency of this molecule class in the regulation of vascular tone and PPAR related gene expression (Omar et al. 1992; Shiraki et al. 2005). For three further lipids a specific identification was not possible but they could be assigned to the lipid classes of diacylglycerol and glycerophosphoinositol isomers. These molecules are part of cell membranes and play a role in signal transduction and fatty acid storage (Romanowicz and Bankowski 2009).

Two additional compounds were putatively identified as the exogenous substances labetalol and terbutalin. Labetalol is used for the treatment of hypertension in pregnant women 
(a) Taurodeoxycholic acid isomer

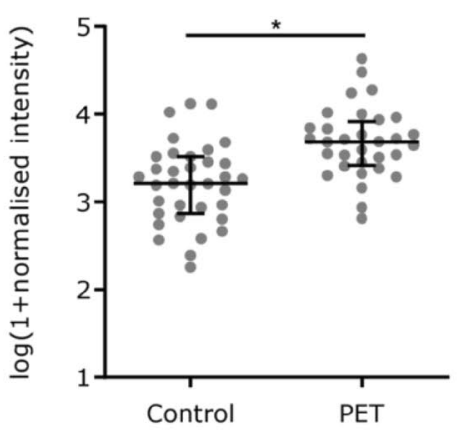

N1-Methyl-pyridone-carboxamide isomer

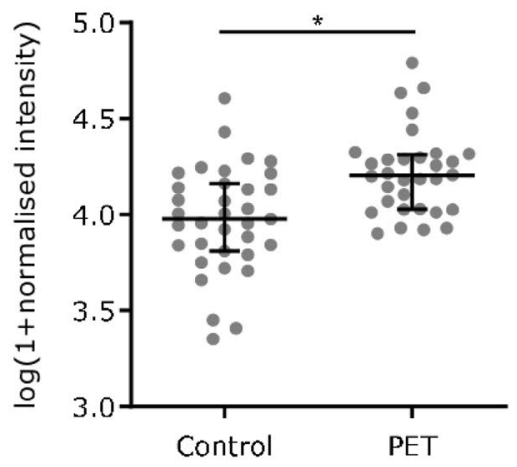

(b)

Hydroxyhexacosanoic acid isomer

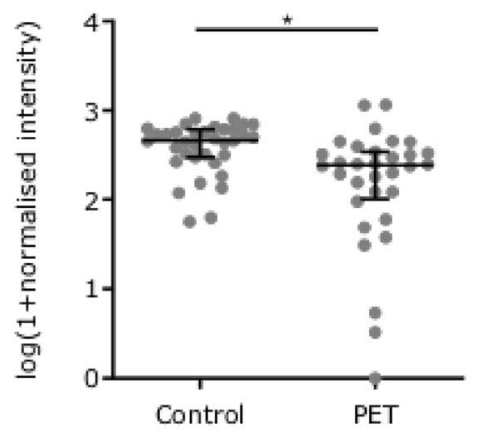

Diacylglycerol isomer (32:1)

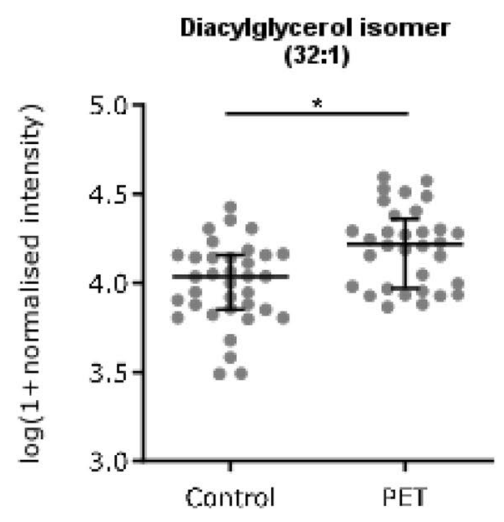

Methionine sulfoxide

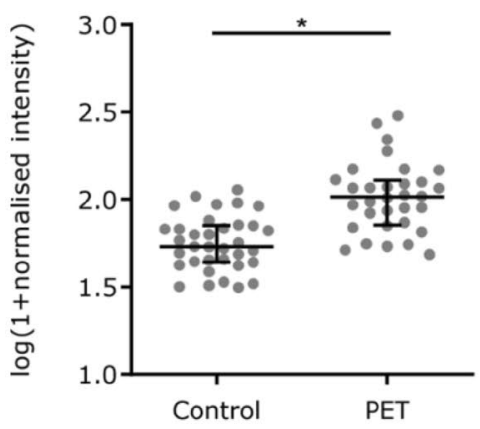

Urocanic acid

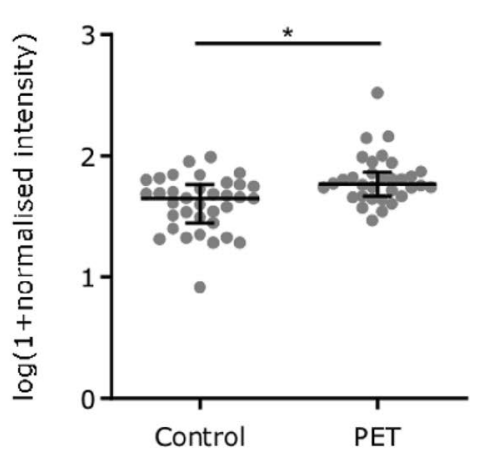

Phosphatidylinositol isomer

(32:1)

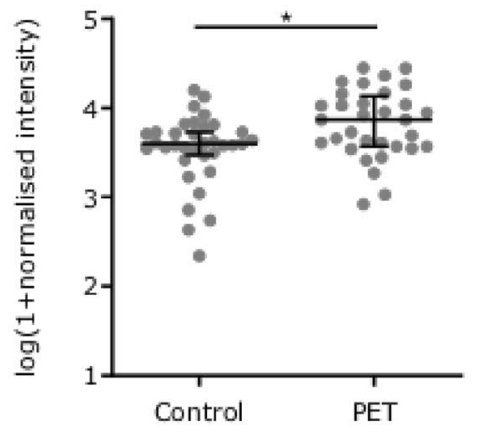

3-Hydroxyanthranilic acid

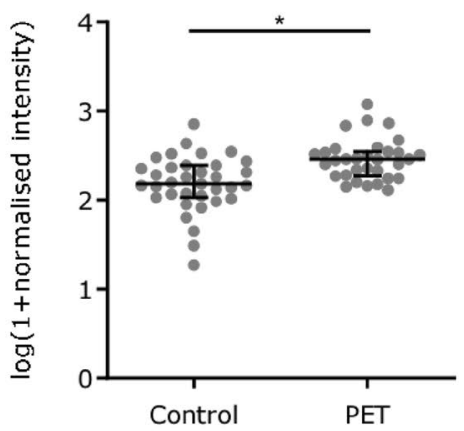

Diacylglycerol isomer (32:2)

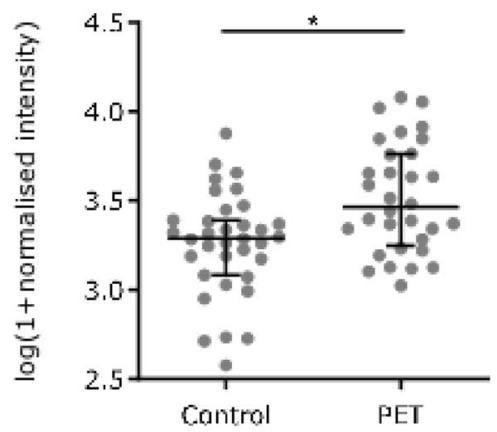


4Fig. 3 Scatter dot plots of potential biomarkers in the polar (a) and apolar (b) phase. All shown biomarkers fulfil criteria of both MVA and UVA. MVA criteria were a VIP value $>1$ in the OPLS-DA model shown in Fig. 1c. Criteria for UVA were a q-value $<0.05(*)$, a fold change $>1.5$ and $\mathrm{CV}(\mathrm{QC})<30 \%$. Dots show $\log (1+$ normalised intensity) of control $(n=35)$ and pre-eclampsia (PET, $n=32)$ samples. Error bars represent median and interquartile ranges. For more details see supplemental data (Tables 1, 2 and Table 3 )

and a number of subjects in the pre-eclampsia cohort were receiving this drug. Terbutalin is a $\beta_{2}$-receptor agonist, used for the management of asthma symptoms or as a tocolytic. The fact that the platform could recognise these compounds provides confidence for the applicability of the experimental approach. It is important to note that metabolomics is sensitive to exogenous influences in that it will also detect exogenous compounds derived from diet, food, lifestyle, drugs or gut microflora.

In summary, this study demonstrates that the metabolome undergoes substantial changes in pre-eclamptic women and a range of novel biomarkers were identified. Of the aforementioned compounds none are known and clinically used biomarkers in pre-eclampsia (Magee et al. 2014). As such, the identified compounds represent novel biomarkers that could help to provide new insights into the ongoing pathophysiological processes of this disease and could eventually lead to the identification of new drug targets. Although most of the putatively identified biomarkers have not been previously described, it was in many cases possible to relate their metabolic pathways to previously published studies as described above.

This work illustrates the potential of untargeted metabolomics in pre-eclampsia research to discover metabolic perturbations in pre-eclampsia potentially leading to novel hypotheses to help understand the underlying aetiology of this disease.

Acknowledgements We thank the patients for participating in this study and the clinical staff of the Department of Obstetrics and Gynaecology at the Royal Derby Hospital for their co-operation.

Author contributions K.N.S.: Sample collection, study design, experiments, data analysis, interpretation, drafting and finalizing article; D.H.K.: Study design, data analysis; C.A.O.: Study design, data analysis; A.Y.W.: Sample collection, study design; D.P.H.: Sample collection, study design; F.B.P.: Study design, interpretation; R.N.K.: Study design, interpretation, drafting and finalizing article; D.A.B: Study design, interpretation, drafting and finalizing article; All authors read and approved the manuscript.

Funding This work was supported by the British Heart Foundation [grant number PG/10/49/28422].

\section{Compliance with ethical standards}

Ethical approval All procedures performed in studies involving human participants were in accordance with the ethical standards of the insti- tutional and/or national research committee and with the 1964 Helsinki declaration and its later amendments or comparable ethical standards. Informed consent was obtained from all individual participants included in the study.

Open Access This article is distributed under the terms of the Creative Commons Attribution 4.0 International License (http://creativeco mmons.org/licenses/by/4.0/), which permits unrestricted use, distribution, and reproduction in any medium, provided you give appropriate credit to the original author(s) and the source, provide a link to the Creative Commons license, and indicate if changes were made.

\section{References}

Bahado-Singh, R. O., Akolekar, R., Mandal, R., Dong, E., Xia, J., Kruger, M., et al. (2012). Metabolomics and first-trimester prediction of early-onset preeclampsia. Journal of Maternal-Fetal and Neonatal Medicine, 25, 1840-1847.

Bahado-Singh, R. O., Akolekar, R., Mandal, R., Dong, E., Xia, J., Kruger, M., et al. (2013). First-trimester metabolomic detection of late-onset preeclampsia. American Journal of Obstetrics and Gynecology, 208, 58.e1-58.e7.

Chan, E. C. Y., Pasikanti, K. K., \& Nicholson, J. K. (2011). Global urinary metabolic profiling procedures using gas chromatographymass spectrometry. Nature Protocols, 6, 1483-1499.

De Oliveira, L., Câmara, N. O. S., Bonetti, T., LoTurco, E. G., Bertolla, R. P., Moron, A. F., et al. (2012). Lipid fingerprinting in women with early-onset preeclampsia: A first look. Clinical Biochemistry, $45,852-855$.

Dunn, W. B., Broadhurst, D. I., Atherton, H. J., Goodacre, R., \& Griffin, J. L. (2011). Systems level studies of mammalian metabolomes: The roles of mass spectrometry and nuclear magnetic resonance spectroscopy. Chemical Society Reviews, 40, 387-426.

Eng, J. (2007). ROC analysis: Web-based calculator for ROC curve. Retrieved February 10, 2018 from http://www.jrocfit.org.

Gardosi, J., Chang, A., Kalyan, B., Sahota, D., \& Symonds, E. M. (1992). Customised antenatal growth charts. Lancet, 339, 283-287.

Gardosi, J., Mongelli, M., Wilcox, M., \& Chang, A. (1995). An adjustable fetal weight standard. Ultrasound in Obstetrics and Gynecology, 6, 168-174.

Huppertz, B. (2008). Placental origins of preeclampsia: Challenging the current hypothesis. Hypertension, 51, 970-975.

Kenny, L. C., Broadhurst, D., Brown, M., Dunn, W. B., Redman, C. W. G., Kell, D. B., et al. (2008). Detection and identification of novel metabolomic biomarkers in preeclampsia. Reproductive Sciences, 15, 591-597.

Kenny, L. C., Broadhurst, D. I., Dunn, W., Brown, M., North, R. A., McCowan, L., et al. (2010). Robust early pregnancy prediction of later preeclampsia using metabolomic biomarkers. Hypertension, 56, 741-749.

Kenny, L., Dunn, W., Ellis, D., Myers, J., Baker, P., \& Kell, D. (2005). Novel biomarkers for pre-eclampsia detected using metabolomics and machine learning. Metabolomics, 1, 227-234.

Kim, D. H., Allwood, J. W., Moore, R. E., Marsden-Edwards, E., Dunn, W. B., Xu, Y., et al. (2014). A metabolomics investigation into the effects of HIV protease inhibitors on HPV16 E6 expressing cervical carcinoma cells. Molecular BioSystems, 10, 398-411.

Kuc, S., Koster, M. P. H., Pennings, J. L. A., Hankemeier, T., Berger, R., Harms, A. C., et al. (2014). Metabolomics profiling for identification of novel potential markers in early prediction of preeclampsia. PLoS ONE, 9, e98540. 
Lisonkova, S., \& Joseph, K. S. (2013). Incidence of preeclampsia: Risk factors and outcomes associated with early- versus late-onset disease. American Journal of Obstetrics and Gynecology, 209, 544. e1-544.e12.

Magee, L. A., Pels, A., Helewa, M., Rey, E., von Dadelszen, P., \& Canadian Hypertensive Disorders of Pregnancy Working Group. (2014). Diagnosis, evaluation, and management of the hypertensive disorders of pregnancy: Executive summary. Journal of Obstetrics and Gynaecology Canada, 36, 416-441.

Mashima, R., Nakanishi-Ueda, T., \& Yamamoto, Y. (2003). Simultaneous determination of methionine sulfoxide and methionine in blood plasma using gas chromatography-mass spectrometry. Analytical Biochemistry, 313, 28-33.

Meyer, N. L., Mercer, B. M., Friedman, S. A., \& Sibai, B. M. (1994). Urinary dipstick protein: A poor predictor of absent or severe proteinuria. American Journal of Obstetrics and Gynecology, 170, 137-141.

Murphy, M. S., \& Smith, G. N. (2016). Pre-eclampsia and cardiovascular disease risk assessment in women. American Journal of Perinatology, 33, 723-731.

Nilsen, R. M., Bjorke-Monsen, A. L., Midttun, O., Nygard, O., Pedersen, E. R., Ulvik, A., et al. (2012). Maternal tryptophan and kynurenine pathway metabolites and risk of preeclampsia. Obstetrics and Gynecology, 119, 1243-1250.

Odibo, A. O., Goetzinger, K. R., Odibo, L., Cahill, A. G., Macones, G. A., Nelson, D. M., et al. (2011). First-trimester prediction of preeclampsia using metabolomic biomarkers: A discovery phase study. Prenatal Diagnosis, 31, 990-994.

Omar, H. A., Figueroa, R., Omar, R. A., \& Wolin, M. S. (1992). Properties of an endogenous arachidonic acid-elicited relaxing mechanism in human placental vessels. American Journal of Obstetrics and Gynecology, 167, 1064-1070.

Orczyk-Pawilowicz, M., Jawien, E., Deja, S., Hirnle, L., Zabek, A., \& Mlynarz, P. (2016). Metabolomics of human amniotic fluid and maternal plasma during normal pregnancy. PLoS ONE, 11, $\mathrm{e} 0152740$.

Raff, A. C., Meyer, T. W., \& Hostetter, T. H. (2008). New insights into uremic toxicity. Current Opinion in Nephrology and Hypertension, 17, 560-565.

Ravipati, S., Baldwin, D. R., Barr, H. L., Fogarty, A. W., \& Barrett, D. A. (2015). Plasma lipid biomarker signatures in squamous carcinoma and adenocarcinoma lung cancer patients. Metabolomics, 11, 1600-1611.

Raymond, D., \& Peterson, E. (2011). A critical review of early-onset and late-onset preeclampsia. Obstetrical \& Gynecological Survey, 66, 497-506.
Redman, C. W. (1991). Current topic: Pre-eclampsia and the placenta. Placenta, 12, 301-308.

Romanowicz, L., \& Bankowski, E. (2009). Lipid compounds of the umbilical cord artery and their alterations in preeclampsia. Atherosclerosis, 204, e44-e51.

Rutkowski, B., Slominska, E., Szolkiewicz, M., Smolenski, R. T., Striley, C., Rutkowski, P., et al. (2003). N-methyl-2-pyridone5-carboxamide: A novel uremic toxin? Kidney International Supplements, 63, S19-S21.

Shiraki, T., Kamiya, N., Shiki, S., Kodama, T. S., Kakizuka, A., \& Jingami, H. (2005). Alpha, beta-unsaturated ketone is a core moiety of natural ligands for covalent binding to peroxisome proliferatoractivated receptor gamma. Journal of Biological Chemistry, 280, 14145-14153.

Steegers, E. A., von Dadelszen, P., Duvekot, J. J., \& Pijnenborg, R. (2010). Pre-eclampsia. Lancet, 376, 631-644.

Sumner, L. W., Amberg, A., Barrett, D., Beale, M. H., Beger, R., Daykin, C. A., et al. (2007). Proposed minimum reporting standards for chemical analysis Chemical Analysis Working Group (CAWG) metabolomics standards initiative (MSI). Metabolomics, 3, 211-221.

Sumner, L. W., Lei, Z. T., Nikolau, B. J., Saito, K., Roessner, U., \& Trengove, R. (2014). Proposed quantitative and alphanumeric metabolite identification metrics. Metabolomics, 10, 1047-1049.

Turner, E., Brewster, J. A., Simpson, N. A. B., Walker, J. J., \& Fisher, J. (2008). Aromatic amino acid biomarkers of preeclampsia-a nuclear magnetic resonance investigation. Hypertens Pregnancy, 27, 225-235.

Waters Corporation (2015) Progenesis QI User Guide 2.0.

WHO (2015) World Health Statistics 2015.

Worton, S. A., Greenwood, S. L., Wareing, M., Heazell, A. E., \& Myers, J. (2019). The kynurenine pathway; A new target for treating maternal features of preeclampsia? Placenta, 84, 44-49.

Wu, P., van den Berg, C., Alfirevic, Z., O'Brien, S., Röthlisberger, M., Baker, P. N., et al. (2015). Early pregnancy biomarkers in preeclampsia: A systematic review and meta-analysis. International Journal of Molecular Sciences, 16, 23035-23056.

Zelena, E., Dunn, W. B., Broadhurst, D., Francis-McIntyre, S., Carroll, K. M., Begley, P., et al. (2009). Development of a robust and repeatable UPLC-MS method for the long-term metabolomic study of human serum. Analytical Chemistry, 81, 1357-1364.

Publisher's Note Springer Nature remains neutral with regard to jurisdictional claims in published maps and institutional affiliations. 